\title{
A controlled trial of the use of dip slides in general practice for the diagnosis of urinary infection
}

\author{
ROSALIND MASKELL
}

\author{
From the Public Health Laboratory and Wessex Renal Unit, St Mary's General Hospital, Portsmouth
}

SYNOPSIS A controlled trial of the use and interpretation of dip-inoculation slides for the diagnosis of urinary infection in four general practices is reported. Two slides were inoculated from each specimen, one being incubated and read in the surgery by the practitioner, the other being sent to the laboratory for incubation and interpretation. It is shown that the presence or absence of bacteriuria can be detected with a high degree of accuracy by a surgery procedure. The implications for the laboratory, the patient, and the practitioner are discussed. It is suggested that the financial saving on laboratory time and unnecessary treatment would far outweigh the outlay on apparatus and materials, and that many patients would benefit from the accurate diagnosis of urinary infection which, for a variety of reasons, is at present denied them.

It is now accepted that urinary infection cannot be diagnosed on clinical grounds alone (Gallagher, Montgomerie, and North, 1965; Steensberg, Bartels, Bay-Nielsen, Fanøe, and Hede, 1969), and that culture of a fresh, carefully collected midstream urine is essential. The implications of this, both for general practitioners and for laboratories, are considerable. Symptoms suggesting urinary infection are thought to account for $1 \%$ of consultations in general practice (British Medical Journal, 1969), and adequate laboratory cover for this is impossible. For a variety of reasons, these patients are usually treated with antibiotics, although probably less than half of them have bacterial infection, and it has been shown that antibiotics do not influence the disappearance of symptoms in patients without bacteriuria (Maskell and Polak, 1972). Many practitioners work at a distance from laboratories (the area served by this laboratory is 168 square miles) and find it difficult to send fresh midstream urines for culture. In recent years this difficulty has been partly overcome by the development of dip-inoculation devices (Mackey and Sandys, 1965; Guttmann and Naylor, 1967), but even when these are used and sent to the laboratory for incubation, which is essential (Maskell and Polak, 1970; Mann and Sandys, 1970), there is likely to be a delay of some two to four days before a written report is received. Treatment cannot be withheld for that length of time, and the bacteriological report is usually only of retrospective interest.

Received for publication 20 December 1972.
Clearly there would be many advantages if a dip slide could be inoculated and incubated in the surgery and read by the practitioner the next day. If he could diagnose the presence or absence of bacteriuria, treatment could be withheld or stopped in those patients without infection (negative slides); doubtful and positive slides could be sent to the laboratory for interpretation and sensitivity testing, and treatment with an antibiotic likely to be appropriate could be started.

With this in mind, a study was made of the various dip inoculation devices in current use, and none of them was thought to be ideal for this purpose. The small size of the Mackey spoon is a disadvantage to the untrained observer, and all the commercially produced dip slides on the market at that time were coated with different media on the two sides, thereby making interpretation more difficult. Several manufacturers were approached, and one (Oxoid Ltd) agreed to make a slide covered on one side only with CLED (cystine-lactose-electrolyte-deficient) agar which is accepted as a suitable single culture medium for urine, and to supply them for a controlled trial of their use and interpretation in general practice.

\section{Methods}

Four general practices of different types were selected: one rural, one in a large council estate, one in a health centre, and one mixed urban and residential. Incubators were installed in the surgeries, and dip slides were provided, together with an inter- 
pretation chart and instructions for collecting midstream urines. None of the practitioners attended the laboratory for any bacteriological instruction beforehand, but a very brief surgery demonstration was given to each, and the importance of careful collection of specimens was emphasized. Scalded-out jam jars were suggested as receptacles, and two slides were dipped by the patient in each midstream urine, one being incubated and read by the practitioner and the other taken to the laboratory either by the patient or by the laboratory specimen collection service for incubation and interpretation.

The criteria used in the laboratory were that growths of less than $10^{3}$ organisms $/ \mathrm{ml}$ were considered negative, $10^{3}-10^{5} / \mathrm{ml}$ doubtful, and $10^{5} / \mathrm{ml}$ or greater positive. Mixed growths were considered doubtful and repeat specimens were requested. Bacterial counts were calculated from charts supplied by the manufacturers. The practitioner read each slide as positive, doubtful, or negative, and recorded his findings on cards which were posted to the laboratory for comparison. No screening of asymptomatic patients was undertaken during the trial, and slides were used only when culture of a midstream urine was an appropriate diagnostic or follow-up procedure.

\section{Results}

The trial lasted from January to June 1972, and the surgery and laboratory interpretations of 423 slides were compared. The findings are shown in the table.

\begin{tabular}{llcc}
\hline Laboratory & \multicolumn{3}{l}{ General Practitioner } \\
\cline { 2 - 4 } & Positive & Doubtful & Negative \\
\hline Positive & 87 & 5 & 9 \\
Doubtful & 15 & 28 & 20 \\
Negative & 11 & 22 & 226 \\
\hline
\end{tabular}

Table Comparison of general practitioner and

laboratory interpretation of 423 slides

ERRORS IN SURGERY INTERPRETATION

For the purpose of the study, which was to assess whether practitioners could differentiate negative slides from positive and doubtful ones, a practitioner was considered to have made an error if he read a slide as negative which the laboratory considered positive or doubtful. Twenty-nine such errors were made ( $6.8 \%$ of the total). Of these 29 slides, only nine $(2 \cdot 1 \%)$ were read as positive in the laboratory, the remainder being interpreted as doubtful.

INCIDENCE OF POSITIVE SLIDES

One hundred and one slides were read as positive by the laboratory $(23.8 \%): 87$ of these were also considered positive by the practitioners, five doubtful, and nine negative.

\section{INCIDENCE OF NEGATIVE SLIDES}

Two hundred and twenty-six slides $(54 \%$ ) were read as negative by the laboratory and the practitioners. A further 33 slides were considered negative by the laboratory, but either positive or doubtful by the practitioners. Twenty-four of these were read in the first three months and nine in the second three months, and it seems possible that this is accounted for by the increasing ability of the practitioners to differentiate between infection and contamination.

\section{DOUBTFUL SLIDES}

Sixty-three slides were read as doubtful by the laboratory. As the likely explanation for this high figure was that these midstream urines were contaminated, the percentage of doubtful slides was analysed separately for the individual practices. Two practices had been using a dip-inoculation technique for urine culture for some years and had considerable experience of the problem of instructing patients in the careful collection of midstream urine. The percentages of doubtful slides from these practices was $12 \cdot 1$ and 14. The other two practices had had no previous experience of dip inoculation, and their percentages were 19.4 and 14 . The differences are not statistically significant.

It seemed possible that, even within the short duration of this study, the collection technique for midstream urine improved, because the overall percentage of doubtful slides for the first three months was 17.6 (41 out of 233) and fell to 11.5 (22 out of 190) in the second three months. This difference, however, is not statistically significant $(\mathrm{P}=0 \cdot 12)$.

\section{Discussion}

These results show clearly that the presence or absence of bacteriuria can be detected with a high degree of accuracy by a surgery procedure. The small error made by the practitioners is clearly outweighed by the advantages of such a technique over the situation which exists in the vast majority of areas at present. A few doctors practise within reach of laboratories which take a particular interest in urinary infection and provide collection facilities and rapid reporting of results for midstream urines. However, the majority of practitioners do not have these facilities, and have little option but to treat all patients who have symptoms without bacteriological confirmation of infection. By the use of dip slides, patients who are now repeatedly treated for 
symptoms without bacteriological diagnosis or confirmation of cure, could be accurately diagnosed and identified as requiring further investigation if infection was shown to persist. Those without infection could be saved unnecessary antibiotic therapy. The fact that $54 \%$ of slides in the trial were negative, even though no screening of asymptomatic patients was undertaken, shows that an overnight bacteriological diagnosis could save or shorten many courses of unnecessary chemotherapy and confirm successful treatment of infection, without recourse to the laboratory. The time and expense involved in transport, form filling, and posting or telephoning of reports would be markedly reduced. During the trial, the expense of the incubators and slides was borne by the manufacturers (Oxoid Ltd): since the completion of the trial, those practitioners who have decided to continue using the technique have had to pay for the slides themselves. However, as it is clear that the saving on laboratory time and unnecessary treatment would far outweigh the outlay on apparatus and materials if this technique were to be widely used, it is arguable that the expense should be borne by the National Health Service and not by the practitioner personally.

It is clear that the work involved in culture of midstream urines as a surgery procedure would mean that only those practitioners who are aware of and interested in the problems of urinary infection would undertake it. In such hands it could provide a useful means of improving the diagnosis and management of patients with urinary symptoms, and could also facilitate the screening of populations such as enuretic children and pregnant women. With the advent of health centres well supplied with ancillary staff, the feasibility of such techniques in general practice is greatly increased.

The use of dip slides would not, of course, preclude direct use of the laboratory for microscopy and culture of fresh midstream urines whenever the practitioner felt it was indicated, for example, in patients with haematuria, or those in whom symptoms persisted without bacteriuria. The slides would, in fact, help to identify such patients who require further investigation.

It must be emphasized that the purpose of this study was not to show that bacteriology can properly. be undertaken in the general practitioner's surgery, rather that the presence or absence of infection can be detected, and only those specimens in which there is definite or doubtful infection need be sent to the laboratory for bacteriological diagnosis.

I am grateful to Oxoid Ltd, who supplied the materials; to Mr R. Pollard of the Central Public Health Laboratory, Colindale, for the statistical calculations; to Drs D. Callum, P. Dilnot and partners, P. J. Fawkner-Corbett and partners, and I. A. Ruthven-Stuart, who undertook the trial in their practices; and to Professor A. Polak and Professor F. O'Grady for helpful advice and criticism.

\section{References}

British Medical Journal (1969). Urinary symptoms in general practice. (Leading article). Brit. med. J., 4, 381.

Gallagher, D. J. A., Montgomerie, J. Z., and North, J. D. K. (1965). Acute infections of the urinary tract and the urethral syndrome in general practice. Brit. med. $J$. 1, 622-626.

Guttmann, D., and Naylor, G. R.E. (1967). Dip slide: an aid to quantitative urine culture in general practice. Brit. med. J., 3, 343-345.

Mackey, J. P., and Sandys, G. H. (1965). Laboratory diagnosis of infections of the urinary tract in general practice by means of a dip-inoculum transport medium. Brit. med. J., 2, 1286-1288.

Mann, P. G., and Sandys, G. H. (1970). Detection of bacteriuria. Lancet, 2, 473-474.

Maskell, R., and Polak, A. (1970). Dip-inoculation media. Lancet, 2 , 309.

Maskell, R., and Polak, A. (1972). Bacteriological facilities for the diagnosis of urinary infection in general practice. In Proceedings of the 2nd National Symposium on Urinary Tract Infection, in the press.

Steensberg, J., Bartels, E. D., Bay-Nielsen, H., Fanøe, E., and Hede, T. (1969). Epidemiology of urinary tract diseases in general practice. Brit. med. J., 4, 390-394. 